\title{
Importancia del trabajo pliométrico en la prevención de lesiones en jugadores de fútbol sala. (1) (1) (9)
}

Importance of plyometric work in the prevention of injuries in futsal players.

William Giovanny Arias Granizo. ${ }^{1}$

Recibido: 11-02-2021 / Revisado: 23-02-2021 /Aceptado: 09-03-2021/ Publicado: 05-04-2021

\begin{abstract}
DOI: https://doi.org/10.33262/cienciadigital.v5i2.1531

Introduction. Preventing injuries in athletes and lowering the costs produced by them, continues to be a great concern for all participants in the field of sports training. The aforementioned is also a priority within futsal; The very characteristics of this sport make its players prone to frequent injuries. In this sense, the theoretical-practical experiences have revealed the importance of plyometric exercises not only to improve the physical performance of athletes, but also to prevent injuries. Objective. Systematize the theoretical - methodological elements on the importance of plyometric work for the prevention of injuries in futsal players. Methodology. The research followed a descriptive, non-experimental methodology, with the use of theoretical methods and the RSL (Systematic Literature Review); All of this allowed us to carry out a bibliographic search in notable databases. Results. A total of 42 sources were consulted, in a range between 2010 and 2020, of which, according to inclusion criteria, 15 were considered potentially relevant. Derived from the above, 3 fundamental criteria are established: the importance of plyometric work in the prevention of injuries in futsal; the existing limitations in the Ecuadorian context on research related to this topic; the need to develop training processes to favor the introduction of this effective alternative within futsal training. Conclusions. It is concluded that indeed, the literature consulted confirms the importance of plyometric work for the prevention of injuries, its use being very beneficial in the case of futsal, if it must be meant that to optimize its use it is necessary to have a
\end{abstract}

1 Universidad Central del Ecuador, Quito, Ecuador, wgarias@uce.edu.ec, ID de ORCID: https://orcid.org/0000-0002-3621-440 
coaching staff and players preparations that can introduce plyometric work to prevent injuries that may arise as part of training or competition.

Keywords: Polymetric work, injury prevention, futsal.

\section{Resumen}

Introducción. Evitar las lesiones en los deportistas y bajar los costos producidos por estas, sigue constituyendo una gran preocupación para todos los participantes en el campo del entrenamiento deportivo. Lo señalado también es prioritario dentro del futsal; las propias características de este deporte, hacen que sus jugadores sean proclives a frecuentes lesiones. En este sentido las experiencias teóricas-prácticas han develado la importancia de los ejercicios pliométricos no sólo para mejorar el rendimiento físico de los deportistas, sino también para prevenir lesiones. Objetivo. Sistematizar los elementos teóricos - metodológicos sobre la importancia del trabajo pliométrico para la prevención de lesiones en jugadores de futsal. Metodología. La investigación siguió una metodología descriptiva, no experimental, con la utilización de métodos teóricos y la RSL (Revisión Sistemática de Literatura); todo ello permitió realizar una indagación bibliográfica en bases de datos notables. Resultados. Se logró consultar un total de 42 fuentes, en un rango comprendido entre el 2010 y el 2020, de las cuales, según criterios de inclusión, 15 fueron consideradas potencialmente relevantes. Derivado de lo anterior se establecen 3 criterios fundamentales: la importancia del trabajo pliométrico en la prevención de lesiones en el futsal; las limitaciones existentes en el contexto ecuatoriano sobre investigaciones relacionadas con este tema; la necesidad de desarrollar procesos de capacitación para favorecer la introducción de esta eficaz alternativa dentro del entrenamiento del futsal. Conclusiones. Se concluye que efectivamente, la literatura consultada confirma la importancia del trabajo pliométrico para la prevención de lesiones, resultando muy beneficioso su utilización en el caso del futsal, si se debe significar que para optimizar su empleo se requiere contar con un cuerpo técnico y con jugadores preparados que puedan introducir el trabajo pliométrico para prevenir las lesiones que puedan presentarse como parte del entrenamiento o la competición.

Palabras claves: Trabajo polimétrico, prevención de lesiones, fútbol sala.

\section{Introducción}

El fútbol sala (futsal) en los últimos años ha alcanzado un importante crecimiento, desarrollo y sobre todo una masiva práctica a nivel mundial, tanto es así que ya son más de 12 millones de jugadores en alrededor de 100 países que lo practican (Beato et al., 2017). Solo en España existen más de cien mil jugadores federados y es considerado uno de los deportes más populares (Ayarra et al., 2018). 
Según López, Vivo, Herrero \& Pareja (2019), es considerado un deporte colectivo, requiriendo de grandes habilidades técnico tácticas y físicas; similares o más llamativas que el fútbol convencional, debido a que las acciones se desarrollan a gran intensidad, con grandes cambios de dirección, toma de decisiones rápidas y efectivas para mantener el balón en su posesión (Sekulic, et al., 2019).

Al ser considerado un deporte con un alto nivel de intensidad en el juego, el factor lesional está presente en todo momento. Es así que las lesiones en el futsal son más frecuentes con relación al fútbol 11; entre otros aspectos porque se desarrolla en un terreno de juego más duro, las dimensiones son más pequeñas, el contacto con los rivales es de mayor continuidad y sobre todo las ejecuciones técnico-tácticas son más exigentes (Martinez, Herrero, Lopez, Guillen \& Fernandez, 2017).

Se comparte con Álvarez, Murillo, Giménez \& Manonelles (2016), al señalar que la prevención de lesiones en el futsal es primordial para el cuerpo técnico y directivos de los clubes; indican los autores precedentes que lograr la prevención de lesiones permitiría contar con la mayoría de sus jugadores a lo largo de la temporada, además de evitar gran cantidad de gastos económicos. Coincidimos con Åman et al. (2018), que las lesiones en los jugadores afecta de manera significativa, incluso en algunos de ellos puede provocar el fin de su carrera deportiva. Del mismo modo, autores como Hewett et al. (2016) manifiestan que: las lesiones producen discapacidad parcial o momentánea y consecuencias en el futuro.

En el caso del futsal al estar contemplado como un deporte dinámico, interválico y acíclico con grandes esfuerzos físicos y tiempos cortos de ejecución, las lesiones están presentes no solo en partidos oficiales, sino en las sesiones de entrenamiento (López et al., 2019). Por tal motivo, dentro de la planificación de las sesiones de entrenamiento en el futsal, la preparación física de los jugadores no solo es determinante para alcanzar los mejores resultados deportivos, sino también para la prevención de lesiones.

Explican López et al. (2019), que las principales lesiones en esta disciplina deportiva están relacionadas al sistema músculo-esquelético de los miembros inferiores como: lesiones musculares, ligamentosas, tendinosas y articulares. Este hecho es debido a que en los entrenamientos no se pone de manifiesto la mayor intensidad de las acciones técnico-tácticas y físicas durante su práctica, además de que es más frecuentes cuando el nivel de competencia es más elevado (Pujals et al., 2016).

El ciclo de estiramiento y acortamiento del músculo es un rasgo particular del entrenamiento con ejercicios pliométricos, donde la unidad músculo tendinosa sufre un alargamiento de las fibras, seguido por una contracción concéntrica. Es por ello sustancial considerar que el entrenamiento pliométrico, mejora la capacidad para producir en el menor tiempo posible una mayor cantidad de fuerza máxima (Alfaro, Salicetti, y Jiménez, 2018). Los ejercicios pliométricos además de mejorar la fuerza muscular aportan un mejor control neuromuscular (Tsang \& DiPasquale, 2011). 
Siguiendo este orden de ideas, se coincide en que si bien es cierto que inicialmente los ejercicios pliométricos se utilizaron únicamente para mejorar la condición física de los atletas, hoy en día también han sido empleados para la prevención de lesiones. Estudios realizados demuestran efectos positivos del empleo de los ejercicios pliométricos en la prevención de lesiones del ligamento cruzado anterior (Willadsen, Zahn, \& Durall, 2019). Del mismo modo Reina (2020) manifiesta que: los ejercicios pliométricos pueden brindar de manera eficaz beneficios en la rehabilitación física y la prevención de lesiones.

Para el cuerpo técnico y su equipo multidisciplinar, es necesario reducir el número de lesiones en sus equipos, la planificación del entrenamiento pretende alcanzar objetivos claros y la prevención de lesiones debe ser uno de los factores más importantes (Álvarez et al., 2016). Dentro de la preparación física de los jugadores podemos contar con aportes importantes de los ejercicios pliométricos y sus efectos positivos dentro del rendimiento físico como en la prevención de lesiones.

Independientemente de lo expuesto se debe significar que, debido a la falta de conocimientos por parte de entrenadores y preparadores físicos en cuanto a las vías o alternativas a utilizar para la prevención de lesiones, se acarrean consecuencias negativas, muchas de las cuales están relacionadas con índices altos de lesiones. Como es lógico lo anteriormente explicado incide negativamente en el proceso de entrenamiento y de preparación de los jugadores y por supuesto en el resultado deportivo. Como bien señala Mueller-Wohlfahrt et al. (2013), al no poder contar con la plantilla completa de jugadores durante las competencias a lo largo del año o temporada se provocan consecuencias económicas de gran impacto para el club, los cuales comprometen el desempeño y resultados deportivos. De todo ello tampoco escapa el futsal.

Es transcendental por tanto comprender los múltiples beneficios que ofrecen los ejercicios pliométricos para la prevención de lesiones. Las investigaciones teóricas unidas a las experiencias prácticas han venido develando que el trabajo pliométrico es empleado para mejorar la condición física, optimizando las características o patrones de habilidades en los jugadores y también como una importante alternativa para la prevención de lesiones (Rezaimanesh, Amiri \& Saidian, 2011).

Sin embargo, lo planteado aun no alcanza la importancia requerida sobre todo en el contexto ecuatoriano, donde son pocos los especialistas y entrenadores de futsal que muestran interés por realizar investigaciones que ayuden a mejorar las condiciones físicas, técnico-tácticas, psicológicas y teóricas de los deportistas y a su vez prevenir lesiones.

Por lo expuesto, el objetivo del estudio está centrado en sistematizar los elementos teóricos - metodológicos sobre la importancia del trabajo pliométrico para la prevención de lesiones en jugadores de futsal. 


\section{Desarrollo}

\subsection{Generalidades del trabajo pliométrico en el entrenamiento del Futsal.}

La pliometría describe una forma rápida de entrenamiento para mejorar la producción de la fuerza, o sea para lograr la capacidad de los músculos para contraerse rápidamente, la cual prioriza ejercicios rápidos, explosivos y potentes.

En este ámbito, debemos tener en cuenta que el principio de la pliometría está basado en un músculo que al principio se estira rápidamente y posteriormente se contrae fuertemente, ello permite un mayor choque de fuerza para la fase siguiente de estiramiento del músculo en movimiento. Específicamente en el caso de los futbolistas, cuanto mejor sea la coordinación de este proceso, más rápido podrá correr el atleta y realizar los diferentes cambios de ritmo dentro de la carrera.

Al respecto, concordamos con Tomalá (2020), al enunciar que mediante los ejercicios pliométricos se logra que el músculo alcance su máximo potencial en un corto período de tiempo, enfatizando que la aplicación en el entrenamiento constituye una vía efectiva para mejorar la fuerza explosiva de los futbolistas y su rendimiento deportivo de manera general.

De igual manera compartimos con Sinovas, Rodríguez \& Cerezal (2020), al afirmar que los ejercicios pliométricos capacitan a los músculos para alcanzar su máximo nivel de fuerza, en un corto período de tiempo; o sea, que constituyen ejercicios que unen fuerza y velocidad en el movimiento para producir potencia.

Al respecto, Hamid, Jaafar \& Ali, (2014), seguido por Simbaña (2018), aseveran que estos ejercicios tienen como objetivo principal el de acortar el tiempo de contracción excéntrica (pliométrico) y el inicio de la contracción concéntrica aplicando la mayor fuerza posible en el menor tiempo posible.

En relación con ello, Muñoz \& Duro (2012), opinan que para que realmente el entrenamiento proporcione resultados de ganancias, se debe prestar especial atención en el período de transición entre las fases excéntricas, conocidas también como fase de amortización, constituyendo esto la clave de este entrenamiento. Señalan además que una fase rápida de amortización (alrededor a una centésima de segundo), permitirá la generación por el cuerpo de una poderosa contracción que es la que producirá esas ganancias de potencia.

Siguiendo este orden de ideas reviste gran importancia la correcta aplicación dentro del entrenamiento de estas fases. Conforme a Lagos (2019), conocer los factores de rendimiento del fútbol sala será uno de los primeros pasos para programar los contenidos de entrenamiento, los valores óptimos a alcanzar y de forma conjunta, formar parte de las estrategias preventivas.

Son varios los autores que han estudiado el rendimiento relacionado con la aplicación del trabajo pliométrico en el futbol sala, entre ellos podemos mencionar a Karavelioglu et al. 
(2016); Neves da Silva et al. (2017), quienes opinaron que se ha observado que el entrenamiento pliométrico puede ser efectivo para la mejora de la explosividad y aceleración con intervenciones entre cuatro y ocho semanas.

Un aspecto adicional a la aplicación del trabajo pliométrico en el fútbol sala comparado a otros deportes, lo constituyen las propias particularidades de este deporte, en el cual de manera preponderante abundan las diferentes acciones de intensidad y cambios de ritmo en las velocidades de desplazamiento que requiere, elevando en un alto grado la necesidad de incluir una correcta aplicación durante los entrenamientos de manera que se obtengan los resultados esperados. Convenimos con Beato et al. (2016); Naser et al. (2017), en que el fútbol sala es un deporte de acciones intermitentes a alta intensidad con constantes cambios de dirección.

En esta dirección, se reafirma la importancia que reviste la aplicación de la pliometría en el deporte de fútbol sala. Respecto a ello, Noguera \& Gamboa (2018) explicaron que la pliometría, es un método positivo para aplicarlo al deporte de fútbol sala, ya que este deporte es explosivo en donde se necesita gran potencia y velocidad para las rápidas acciones de juego que se presentan durante un partido.

De igual manera García \& Peña (2016), han señalado que la inclusión de la pliometría en este deporte ayuda a mejorar tanto la velocidad, que es el factor primordial como otros factores físicos. También en esa línea Rodríguez-Gómez, Merchan \& Forero (2014), han mencionado que el entrenamiento con ejercicios pliométricos mejora la velocidad y la fuerza y se puede utilizar para obtener resultados rápidos en periodos de tiempo cortos.

En sentido general, lo anteriormente expuesto, afianza la importancia de la aplicación del trabajo pliométrico en los entrenamientos del Fútbol Sala. Su empleo resulta una alternativa que evidentemente favorece el rendimiento deportivo de los jugadores y a su vez contribuye a la prevención de lesiones durante los entrenamientos y las competiciones.

\subsection{El trabajo pliométrico y su incidencia en la prevención de lesiones.}

Como se conoce, mantener un nivel competitivo estable con óptimos resultados a lo largo de un ciclo o temporada deportiva, sólo se alcanza a través de la aplicación de estrategias de entrenamiento que combinen de manera efectiva el logro de un rendimiento óptimo y el mantenimiento de un estado físico adecuado. Lo anteriormente señalado implica prevenir la disminución de lesiones, de modo tal que permita a los deportistas estar aptos para los resultados esperados durante todo el ciclo o temporada de preparación y competencias.

En esta dirección coincidimos con Eckard, Padua, Hearn, Pexa \& Frank (2018), al plantear que dentro de los dos principales objetivos del entrenamiento, además de optimizar el rendimiento, se encuentra lograr reducir el número y severidad de las 
lesiones, de manera que los deportistas dispongan del mayor tiempo posible, en óptimas condiciones físicas, durante el período competitivo. Por otra parte, también remarcaron que la creación de picos de entrenamiento (elevación o disminución repentinas de las cargas) suelen ser favorables a la aparición de lesiones en los deportistas.

En el caso del fútbol sala, como habíamos expresado, primeramente, dado su particularidad reviste de gran importancia la especificidad propia de su entrenamiento. Acorde a como indica Lagos (2019), el conocer los factores de rendimiento del fútbol sala, será uno de los pasos iniciales a tener en cuenta en la programación de los contenidos del programa de entrenamiento, con vistas a alcanzar los valores óptimos y conjuntamente elaborar las estrategias preventivas para evitar las lesiones.

Son varios los autores que al respecto se han referido a la incidencia que tiene la aplicación de los ejercicios pliométricos en los entrenamientos en relación a la conjugación del logro de rendimiento y prevención de lesiones en los deportistas, especialmente en el fútbol sala. Al respecto, se concuerda con Reina (2020), al manifestar que el ejercicio pliométrico desarrolla la capacidad del deportista de asimilar los diferentes cambios de posición y ritmo en el movimiento, los cuales mejoran los niveles de adaptabilidad osteomioarticular y disminuyen las lesiones, debido al incremento de la capacidad de trabajo propioceptivo a nivel de las estructuras anatómicas del tren inferior.

En esta misma dirección Sadoghi, Keudell \& Vavken (2012), en su estudio de metaanálisis realizado, mostraron la efectividad del entrenamiento neuromuscular basado en la aplicación de ejercicios pliométricos para la prevención de lesiones a nivel del tren inferior, el cual arrojó que, del total de muestra consultada, se produjo un $85 \%$ de efectividad en los deportistas hombres y un $52 \%$ en mujeres.

Un aspecto de vital importancia lo constituye el conocimiento de las particularidades propias del fútbol sala, dado la intensidad física con que se realiza y las propias exigencias físicas que requiere su práctica, en este sentido coincidimos con el resultado de los estudios realizados por Beato, Coratella, Schena \& Hulton (2017), donde explican la importancia del concocimiento de la información sobre cargas mecánico-musculares, tales como, la distancia total recorrida (TD), poder metabólico (MP), potencia metabólica de alta intensidad (HDMI) y la carga dinámica del cuerpo (DBL), aspectos que deben tener en cuenta los entrenadores al aplicar el entrenamiento.

En este ámbito se han realizado otros estudios por diferentes autores, en los cuales se han obtenido resultados alentadores en relación al rendimiento y la prevención de lesiones. En este caso podemos citar a (Neves da Silva et al., 2017), quienes obtuvieron rendimientos significativos en la capacidad de sprint repetidos (RSA), así como la utilización de intervalos de alta intensidad del entrenamiento (HIIT) incluyendo 
intervalos de carrera con lanzaderas de balones (SRIT), el cual mejoró tras cinco semanas de entrenamiento específico con cambios de dirección en jugadoras brasileñas.

De igual manera Ayala, Sainz DeBaranda \& De Ste Croix (2010), obtuvieron confortadores resultados en un equipo español, mediante el empleo de la prueba de resistencia intermitente y las rutinas de estiramiento estático, siendo eficaz para el aumento del rango del movimiento (ROM) de cadera, tanto flexión como extensión, realizado tras 8 semanas de intervención con 6 series x 30 segundos de estiramiento activo durante tres días a la semana.

En relación a ello, los propios autores expresaron que de las diversas técnicas de estiramiento, ya sea la estática, dinámica, balística y la de facilitación neuromuscular propioceptiva (FNP), en el ámbito del deporte y del futbol sala, la que más se utiliza son el estiramiento estático, dinámico y balístico. Por otra parte, las rutinas de estiramiento estático son las que habitualmente los preparadores físicos la prescriben para su ejecución en el calentamiento, debido a que implica un mínimo riesgo de lesión, es eficiente, requiere de escasa asistencia y es eficaz para el aumento del rango del movimiento (ROM).

Al respecto Vargas (2019), plantea que los ejercicios pliométricos además de fortalecer los músculos debido a que aumentan su potencia muscular donde se desarrolla la fuerza, el equilibrio y la coordinación, también mejora el sprint, que contiene a cualidades fundamentales del fútbol sala tales como: la aceleración y la velocidad. Dado que, al realizar los ejercicios pliométricos, se fortalece de hecho, toda la musculatura del miembro inferior, lo que hace que el jugador sea menos propenso a la aparición de cualquier tipo de lesión, aunque la realización de los mismos debe ser de manera correcta, conforme a la metodología y sin forzar el organismo, todo ello origina que el jugador tenga un estado físico corporal más resistente dentro de la cancha.

En analogía con estos mismos aspectos, Acosta, Cetina, Ramírez \& Montealegre-Mesa (2019), señalan que la prevención de lesiones en el jugador de fútbol, es una estrategia de abordaje fundamental, dado que está relacionada con las consecuencias negativas que tiene, tanto para su rendimiento deportivo como para la salud del jugador durante toda su carrera deportiva. Enfatizan los autores precedentes en la necesidad de trazar una correcta estrategia ya que esto contribuye a que se acelere el proceso de recuperación funcional del jugador para su incorporación al terreno de juego, con mejores condiciones físicas.

Estos mismos autores hacen alusión a los principales programas preventivos reconocidos por su especificidad y otros desarrollados por la Federación Internacional de Fútbol Asociado FIFA, entre los que se encuentran: FIFA 11, que conforme a Nouni-García et al. (2018), es un programa de 10 ejercicios, que tiene como objetivos principales, la estabilización lumbo-pélvica o central, ejercicios pliométricos, agilidad y control 
neuromuscular; FIFA 11+, que según Bizzini et al. (2013), es un programa de calentamiento y preventivo de lesiones neuromusculares, con ejercicios de carrera a baja intensidad, fuerza, pliometría y equilibrio así como ejercicios de carrera combinados con movimientos propios del fútbol.

Lo cierto es que tanto Van Beijsterveldt et al. (2012), como Ayala et al. (2017), destacan la importancia de la eficacia para el entrenamiento y acondicionamiento de la mejora en fuerza y equilibrio muscular alrededor de la articulación de la rodilla y su práctica a modo de calentamiento, enfatizando que esto es requerido por lo menos dos veces por semana durante tres meses consecutivos. El FIFA 11 + Shoulder, que es derivado del anterior programa y que conforme Ejnisman et al. (2016), en el mismo los ejercicios se centran en estabilidad del core y control neuromuscular, la fuerza de mecanismo excéntrico y agilidad.

De igual manera y en correspondencia con lo anterior, Romero et al. (2017), reafirman en su estudio la obtención de resultados alentadores relacionados con la disminución de las lesiones resaltando que los programas de prevención aplicados, incluyen el trabajo de la fuerza neuromuscular, estiramientos, pliometría, equilibrio, core y corrección de la técnica de amortiguación del salto y los cambios de dirección.

Otros autores han elaborado y aplicado programas similares de prevención de lesiones, aunque con otros grupos poblacionales, que han servido para comparar resultados, tal es el caso de Saiz (2018), quien afirma que en referencia al programa de entrenamiento elaborado, la clave del mismo se basó en la realización de un calentamiento general y específico muy bien controlado, la realización de estiramientos antes y después del entrenamiento, la utilización de una progresión controlada de la intensidad de los ejercicios variando el contenido de las sesiones y no incluyendo en ningún momento elementos no competitivos durante las mismas. Precisamente, determinó la fiabilidad del programa la relativamente baja incidencia en lesiones y en síntomas músculoesqueléticos durante el entrenamiento.

De la misma manera, se ha constatado que además el entrenamiento con ejercicios pliométricos ejerce una incidencia sobre la propia habilidad del deportista, dado que le permite realizar con una mayor eficacia y seguridad algunos movimientos que antes no podía realizar. En referencia a ello, Mesfin, Sangeeta \& Molla (2015), determinaron en su estudio, que los efectos de este tipo de ejercicios de entrenamiento, permitieron obtener mejoras significativas en determinadas variables de aptitud física y habilidad futbolística de los deportistas, lo que representó un efecto positivo en el dribleo en correlación con la velocidad y agilidad, así como mejoró la capacidad de patear. Estos autores concordaron con Roopchand-Martin \& Lue-Chin (2010), al indicar que el entrenamiento pliométrico se usa ampliamente en acondicionamiento, entrenamiento de potencia y en la prevención 
y rehabilitación de lesiones en algunos deportes y en gran medida en el fútbol y fútbol sala.

Todo lo explicado hace que se coincida con los estudios y autores precedentes al reconocer la importancia que tiene el trabajo pliométrico para la prevención de lesiones en los diferentes deportes, específicamente en el futsal; somos del criterio que al lograr un correcto proceso de entrenamiento donde se haga uso del trabajo pliométrico se podrá prevenir la presencia de lesiones y por consiguiente mejorar los indicadores de rendimiento y resultados deportivos.

\section{Metodología}

La investigación siguió una metodología descriptiva no experimental, con la utilización de métodos teóricos, dentro de estos: el histórico-lógico, el analítico - sintético, inductivo - deductivo y la RSL (Revisión Sistemática de Literatura), todo ello permitió realizar una indagación bibliográfica en bases de datos notables en español e inglés, dentro de las cuales se citan: Dialnet, Scopus, PubMed, Sport Discus, Índices CSIC, Google Académico, Scand J Med Sci Sports, The American Journal of Sports Medicine, J Strength Cond Res. Para encontrar información adicional se realizó una búsqueda en los repositorios nacionales e internacionales que contemplan Tesis de Doctorado y Maestría relacionadas con el tema de investigación.

La estrategia de indagación seguida facilitó tener información confiable y de calidad e identificar en las diferentes bases de datos los artículos e investigaciones potenciales. De igual manera se logró ir limitando la población a consultar según las palabras claves previamente determinadas: trabajo pliométrico, prevención de lesiones, fútbol sala.

Criterios de inclusión

1. Tipo de estudios: Estudios de revisiones teóricas o experimentales que sistematicen o desarrollen teorías en relación con el trabajo pliométrico y su incidencia en la prevención de lesiones, con especial interés en jugadores de fútbol sala.

2. Tipo de participantes: entrenadores y jugadores de fútbol sala.

3. Tipo de resultados: Programas, Metodologías, Modelos, relacionados con el trabajo pliométrico y su incidencia en la prevención de lesiones en jugadores de fútbol sala.

4. Tesis de Doctorado o Maestría realizadas en los 10 últimos años en universidades Internacionales o Nacionales relacionadas con el tema. 
1. Estudios o investigaciones realizadas en un rango de tiempo diferente al contemplado en la investigación y que no aporten elementos significativos para nuestro estudio.

2. Artículo en idioma diferente del español o inglés.

3. Otros estudios que por su tema o resultados no aporten elementos significativos para nuestra investigación

Evaluación de la calidad

La calidad se determinó mediante el seguimiento riguroso de los criterios de inclusión y exclusión establecidos previamente.

\section{Análisis y discusión de resultados en cuanto a bases de datos consultadas:}

Según las bases de datos consultadas y considerando el rango de tiempo establecido, el cual estuvo comprendido entre el 2010 y el 2020, se consultaron un total de 41 fuentes, determinándose como estudios potencialmente relevantes 15 investigaciones, de las cuales, el 100\%, maneja las variables objeto de investigación: trabajo pliométrico y prevención de lesiones, futsal. Se debe significar que, dentro de la Revisión Bibliográfica realizada, se consultaron además Tesis de Doctorado, Maestría y Licenciatura, relacionadas con el tema, los cuales permitieron sistematizar las ideas y teorías precedentes e ir conformando nuestros aportes.

Emanado de lo anterior los resultados evidencian que los años 2018 y 2019, resultaron ser los años donde se ha logrado tener mayor producción científica relacionada con este tema. Destacándose dentro de los autores que han aportado al tema los trabajos realizados por: Alfaro, Salicetti \& Jiménez (2018); Ayarra, Nakamura, Iturricastillo \& Castillo Yanci (2018); Eckard, Padua, Hearn, Pexa \& Frank (2018); Noguera \& Gamboa (2018); Sekulic, Foretic, Gilic, Esco, Hammami, Uljevic, Versic \& Spasic (2019); Vargas (2019). Destacan además autores ecuatorianos que han venido aportando a esta teoría, dentro de los cuales se encuentran Vargas (2019); Simbaña (2018) y Tomalá (2020), sin embargo dada las limitaciones de estudios relacionados con este tema se infiere la necesidad de profundizar en el desarrollo de investigaciones que permitan en primer lugar profundizar en las teorías precedentes en relación con el trabajo pliométrico y su importancia para la prevención de lesiones y en segundo término estudios que puedan demostrar en la práctica dentro del contexto ecuatoriano los impactos de esta novedosa forma de entrenamiento.

A continuación, se exponen en la Tabla No1 los resultados del análisis realizado según criterios de inclusión que permitió determinar investigaciones potenciales 
Tabla No 1 Resultado de Investigaciones potenciales directos para la investigación

\begin{tabular}{|c|c|c|c|}
\hline Autorías, Tit & Base de Datos & Intervención & Conclusiones \\
\hline $\begin{array}{l}\text { Markovic, \& } \\
\text { Mikulic (2010). } \\
\text { Adaptaciones neuro- } \\
\text { musculoesqueléticas } \\
\text { y de rendimiento al } \\
\text { entrenamiento } \\
\text { pliométrico de las } \\
\text { extremidades } \\
\text { inferiores. }\end{array}$ & $\begin{array}{l}\text { School of Kinesiology, } \\
\text { University of Zagreb, Zagreb, } \\
\text { Croatia. Adis Data Information } \\
\text { BV. Magazine Sport Medicine. } \\
\text { October 2010. 40(10):859-95. } \\
\text { DOI: } 10.2165 / 11318370- \\
\underline{000000000-00000 .} \text { Source: } \\
\underline{\text { Pub.Med. }}\end{array}$ & 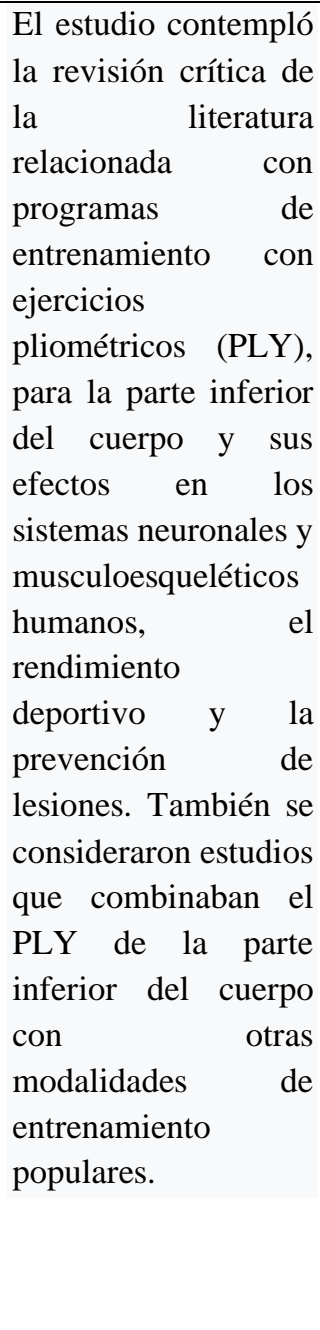 & 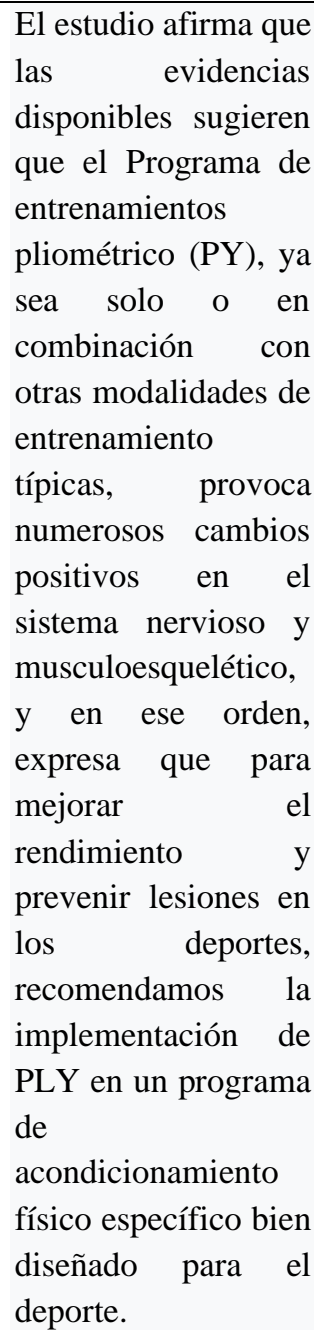 \\
\hline $\begin{array}{l}\text { Muñoz, \& Duro } \\
\text { (2012). El } \\
\text { entrenamiento } \\
\text { pliométrico en el } \\
\text { fútbol } \\
\text { sala: Ganancias de } \\
\text { potencia de salto y } \\
\text { de potencia de } \\
\text { disparo. }\end{array}$ & $\begin{array}{l}\text { EFDeportes.com, Revista } \\
\text { Digital. Buenos Aires- años } \\
\text { 17-No } 169 \text { http:// } \\
\text { www.efdeportes.com }\end{array}$ & $\begin{array}{l}\text { El estudio analiza los } \\
\text { beneficios del } \\
\text { entrenamiento } \\
\text { pliométrico en } \\
\text { jugadores de futbol } \\
\text { sala, tanto para } \\
\text { mejorar la potencia } \\
\text { de disparo, como la } \\
\text { potencia de salto. }\end{array}$ & $\begin{array}{l}\text { Queda demostrado la } \\
\text { indecencia de los } \\
\text { ejercicios } \\
\text { pliométricos durante } \\
\text { el entrenamiento, en } \\
\text { la potencia muscular } \\
\text { en el salto, sin } \\
\text { embargo, } \\
\text { resultados no se } \\
\text { muestran muy } \\
\text { alentadores en lo } \\
\text { ocurre en la potencia } \\
\text { de disparo. }\end{array}$ \\
\hline $\begin{array}{l}\text { Adalid } \\
\text { Propuesta }\end{array}$ & $\begin{array}{r}\text { Retos-Nuevas } \\
\text { en Educación }\end{array}$ & $\begin{array}{l}\text { El trabajo realiza una } \\
\text { aproximación al }\end{array}$ & $\begin{array}{l}\text { La propuesta de } \\
\text { prevención }\end{array}$ \\
\hline
\end{tabular}


incorporación de Física, Deporte y Recreación tareas preventivas 2014, No 26, pp. 163-167. basadas en métodos propioceptivos en fútbol- Universidad de Granada.
Federación Española de Asociaciones de Docentes de Educación Física (FEADEF) ISSN: Edición impresa: 15791726. Edición Web: 1988-2041 (www.retos.org).
Mesfin, Sangeeta \&

Molla

(2015).

Effects of plyometric training on soccer related physical fitness variables of intercollegiate

female soccer players.

\section{estudio de prevención}

mediante la actividad

física, con el objetivo

de identificar las

principales lesiones

para analizar la

incorporación de

medidas que desde el

ámbito de la

preparación física

han demostrado

evidencia de

efectividad en la tarea

preventiva, como son: el entrenamiento de fuerza, de flexibilidad y de propiocepción. incorporó a la

programación anual

entrenamientos

polifacéticos (con

ejercicios

pliométricos, de

fuerza

compensatoria,

estabilización del

core, trabajo

excéntrico $y$

ejercicios de

equilibrio, que

permitieron a los

jugadores alcanzar

niveles más óptimos

en cuanto a carga

muscular se refiere,

cadenas cinéticas

más coordinadas,

mayor tolerancia a la

fatiga $y$ mayor

respuesta ante

desequilibrios

provocados por

cargas externas en la

competición), que

pudieron reducir

significativamente el número de lesiones.

Otros autores han demostrado que la inclusión de este tipo de ejercicios

específicos han

determinado una

menor incidencia

lesional en

comparación con

equipos que no los ha desarrollado.

El programa también

ha mostrado un

cambio significativo en la mejora de las

variables pliométrico en variables de aptitud física relacionadas con las jugadoras de fútbol intercolegiales seleccionadas de

componentes de

aptitud física $\mathrm{y}$

variables de

habilidad 
García Ramos, \&

Peña López (2016).

Efectos de 8

Semanas de

Entrenamiento

Pliométrico y

Entrenamiento

Resistido Mediante

Trineo en el

Rendimiento de

Salto Vertical y

Esprint en

Futbolistas

Amateurs.
Estudio del grupo de Analizó los efectos investigación de análisis de de un entrenamiento rendimiento deportivo de la pliométrico y de un Universidad Central de entrenamiento Cataluña. Barcelona. España.

resistido, mediante arrastres de trineo de 8 semanas de duración sobre el rendimiento en pruebas de salto vertical y velocidad en sprint de 30 metros en futbolistas amateurs.

La investigación verificó los efectos del entrenamiento pliométrico de corta duración (PM) en la composición corporal, flexibilidad y la producción de potencia muscular en atletas de fútbol sala.

\section{futbolística, lo que representó una disminución significativa de las lesiones durante el período.}

Demostró que después de 8 semanas de entrenamiento

pliométrico y de arrastres de trineo en futbolistas, se pudo observar que se producían cambios positivos en el rendimiento de las pruebas de salto pliométrico vertical (ya sea en remates de cabeza o en una acción de salto de un portero). De igual manera, los saltos pliométricos

horizontales parecen incrementar el rendimiento en los sprint, debido a la aplicación horizontal de la fuerza en la carrera.

Los resultados del estudio, muestran que el entrenamiento pliométrico puede ser eficaz para reducir la grasa corporal y aumentar la flexibilidad y la potencia muscular en las atletas de fútbol sala. Por lo tanto, puede sugerir que el entrenamiento

pliométrico puede aplicarse en el campo de la fisioterapia preventiva. 
Beato, Coratella, Biology of Sport, 34(3), 227- El objetivo de este

Schena, \& Hulton 231

doi: estudio

2017). Evaluation

10.5114/biolsport.2017.65998.

of the external \&

internal workload in

female futsal

players.

cuantificar las
Romero, Cuéllar, RICYDE.

González, Bastida, Internacional de Ciencias del

Echarri, Gallardo, Deporte, vol. XIII, núm. 48,

Paredes (2017). abril, 2017, pp. 117-138

Revisión de los Editorial Ramón Cantó Alcaraz factores de riesgo y Madrid, España.

los programas de

prevención de la

lesión del ligamento actividades

locomotoras

mecánicas realizadas durante un partido de fútbol sala femenino, midiendo las diferencias entre la primera y la segunda parte, evaluando sus demandas de carga externa.
El estudio, informa sobre la utilización s de la carga dinámica del cuerpo (DBL),

y durante el análisis de partidos de fútbol sala. Es un parámetro relativamente nuevo que registra cada variación instantánea de actividad y puede contribuir a la investigación de indicadores

específicos de estrés mecánico.

Las investigaciones

futuras pueden analizar mejor su uso en el fútbol sala.

La información sobre cargas mecánicomusculares (por ejemplo, Distancia total recorrida, TD, poder metabólico MP, distancia de potencia metabólica de alta densidad HMD, número de aceleraciones $\quad \mathrm{y}$ desaceleraciones) tiene una importancia crítica en el diseño de protocolos específicos de entrenamiento de fútbol sala de los régimenes de carga y la previsión de lesiones.

Los programas de prevención que han mostrado disminuir la frecuencia lesional inciden en el trabajo del core, la propiocepción, el control motor, el aprendizaje de la 
cruzado anterior en

fútbol femenino:

propuesta de

prevención.
Noguera \& Gamboa (2018). Influencia de la pliometría en la velocidad de desplazamiento de las jugadoras de fútbol sala femenino de la Corporación Universitaria Autónoma del

Cauca.
Corporación Universitaria Autónoma del Cauca. Trabajo de grado para optar al título de profesional en deporte $y$ actividad física. Facultad de Educación Programa de Deporte y Actividad Física. Ciencias aplicadas al deporte y la actividad física.
El estudio tuvo como objetivo determinar la influencia de la pliometría en la velocidad de desplazamiento en jugadoras de fútbolsala femenino. La metodología estuvo enfocada a las ciencias exactas o lo cuantificable, o sea en lo medible, el tipo de investigación fue correlacional porque buscó la relación entre dos variables pliometría y la velocidad de desplazamiento. $\begin{array}{ll}\text { técnica } & \mathrm{de} \\ \text { amortiguación, } & \text { la }\end{array}$

flexibilidad y el trabajo excéntrico. En el estudio se exponen los factores de riesgo específicos a esta lesión a la eficacia mostrada en los diferentes programas preventivos analizados. Los programas de prevención incluyen el trabajo de la fuerza neuromuscular, estiramientos, pliometría, equilibrio, core $\mathrm{y}$ corrección de la técnica de amortiguación del salto y los cambios de dirección.

El estudio validó que el programa de entrenamiento pliométrico para mejorar la velocidad de desplazamiento en la población objeto de estudio, determino que 8 semanas de entrenamiento

influyen

positivamente sobre dicha capacidad. Igualmente que el entrenamiento

pliométrico es un método efectivo de entrenamiento con de resultados a corto plazo, en el que la periodización con cargas selectivas es determinante sobre la incidencia de lesiones en el futsal. 


\begin{abstract}
Simbaña (2018). Los ejercicios pliométricos en la fuerza explosiva del tren Inferior de la selección de fútbol femenino de la Liga Deportiva Parroquial Picaihua
\end{abstract}

$\begin{array}{lrlr}\text { Vargas (2019). } & \text { Proyecto de Investigación } \\ \text { Pliometría en la } & \text { previo a la obtención del título } \\ \text { prevención } & \text { de } & \text { de Licenciada en Terapia Física } \\ \text { lesiones } & & \text { y Deportiva. Universidad } \\ \text { musculotendinosas } & \text { Nacional de Chimborazo. } \\ \text { en futbolistas } & \begin{array}{l}\text { Facultad de Ciencias de la } \\ \text { profesionales }\end{array} & \text { Salud. Carrera de Terapia } \\ & \text { Física y Deportiva. }\end{array}$

Acosta, Cetina,

Ramírez

\&

Montealegre-Mesa

(2019). Programas preventivos, una

estrategia para el

jugador de fútbol.

\section{Tesis de Grado. Universidad Estudió la aplicación} Técnica de Ambato. Ecuador.

.

.

de ejercicios

pliométricos en la

fuerza explosiva del tren inferior de los futbolistas de futsal, a través de un conjunto de ejercicios planificados correctamente.

La investigación tuvo como objetivo demostrar los efectos de los ejercicios pliométricos para prevenir lesiones musculotendinosas en miembros inferiores futbolistas en profesionales, con la particularidad del aumento de su fuerza.
El resultado del estudio aprobó la hipótesis alterna planteada, la cual expresa que si existía relación significativa entre ejercicios pliométricos y fuerza explosiva en los integrantes de la selección de futbol.

Se demostró que los ejercicios

pliométricos tiene efectos positivos durante el entrenamiento

porque mejoro la fuerza y previene lesiones en el tren inferior, estos resultados se refleja en el test de salto horizontal sin carrera tiene una mejoría en un $15 \%$ de la fuerza y el test de salto vertical en un $30 \%$, recalcando que la técnica pliométrica se aplicó al 100\% de la muestra que son futbolistas que pertenecen a la categoría de Reserva del Club Deportivo El Nacional, concluyendo que durante el período de intervención la población no presentó ningún tipo de lesiones musculotendinosas.

Se expuso que un programa preventivo en general estipula el entrenamiento neuromuscular, como herramienta de de prevención de lesiones, como una estrategia de 


\section{Revisión}

bibliográfica.

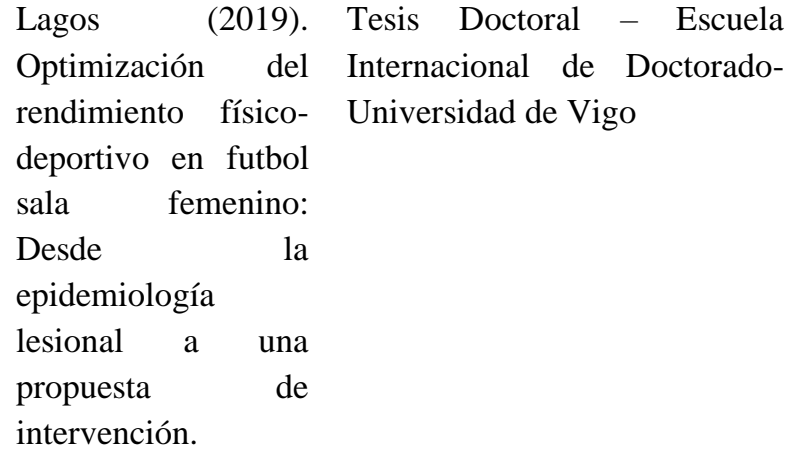

conocimiento específico para el jugador de fútbol. capacidad de generar patrones óptimos de activación muscular, aumentando

estabilidad dinámica articular y patrones de movimiento necesarios; el entrenamiento

propioceptivo $\quad \mathrm{y}$ equilibrio

complementando el control postural evitando desequilibrios en las extremidades inferiores así como la pliometría que mejora la mecánica corporal y reducen lesiones graves, particularmente del ligamento Cruzado Anterior (LCA), teniendo en cuenta, que la rodilla es una articulación de importancia en este deporte y ocupa el $5 \%$ de lesiones que dan en miembros inferiores.

Analiza las diferentes El estudio demostró variables que la escala de relacionadas con la percepción subjetiva optimización del del esfuerzo de la rendimiento físico- sesión (sRPE), deportivo en mostró ser una jugadoras herramienta útil para profesionales de controlar las cargas a fútbol sala femenino. lo largo de una temporada en un equipo de fútbol sala femenino. Las mayores cargas se registraron durante el primer mesociclo, asociado al período preparatorio.

Después, se observó un patrón oscilatorio 
Tomalá

(2020). Tes

Programa

ejercicios

de

pliométricos para el desarrollo de la fuerza explosiva en futbolistas de la categoría sub 16.

de las cargas a lo largo del período competitivo, con cargas de entrenamiento similares, tanto a nivel de microciclo como mesociclo. Además, la distribución de la carga semanal tuvo una tendencia descendiente para que las jugadoras estuviesen plenamente recuperadas el día del partido.

Se realizó un diagnóstico mediante la aplicación de métodos y técnicas empíricas como la revisión documentos, observación encuesta determinar cómo se comporta el empleo de los ejercicios pliométricos para el desarrollo de la fuerza explosiva en futbolistas de la categoría sub 16.

Se realizó un estudio para determinar los niveles de Fuerza Explosiva y mejorar los mismos mediante la aplicación de la Pliometría. Evaluar los niveles de Fuerza Explosiva en el tren inferior, Diseñar $\mathrm{y}$ Plantear un programa de Ejercicios Pliométricos, valorar los resultados postest y analizar los
El estudio constató que mediante los ejercicios

pliométricos se logra que el músculo alcance su máximo potencial en un corto período de tiempo por lo que su aplicación en el entrenamiento constituyen una vía efectiva para mejorar la fuerza explosiva de los futbolistas y su rendimiento

deportivo de manera general

El estudio corroboró que el entrenamiento aplicado sobre los futbolistas del Club deportivo "El

Nacional" trajo consigo efectos significativamente positivos sobre los niveles de saltabilidad entre el pretest y el postest. Tras la implementación del protocolo 


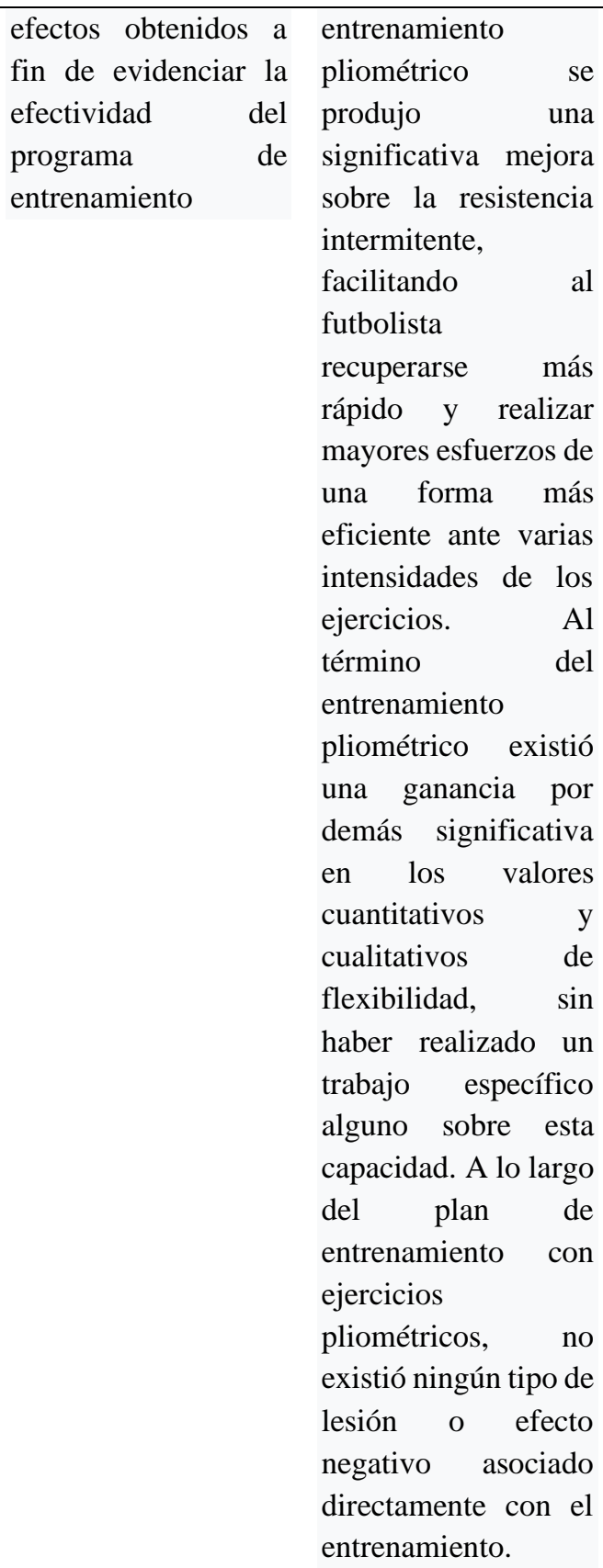




\section{Discusión de Resultados:}

A pesar de que el futsal cuenta con varios años de práctica tanto a nivel internacional como nacional, se puede considerar un deporte joven en comparación con el resto. Como resultado de lo anterior, en relación a este deporte, predominan principalmente los estudios relacionados con el rendimiento deportivo y existe un escaso porcentaje de estudios relativos a la prevención de lesiones en los deportistas.

De igual manera y en concordancia con lo anteriormente expuesto, la aplicación en los entrenamientos de futsal de ejercicios pliométricos para elevar el desempeño de los futbolistas es relativamente nuevo así como la incidencia que sobre la prevención de lesiones trae consigo este tipo de ejercicios.

Ello implica que la aplicación de programas de entrenamiento que incluyan ejercicios pliométricos, que fortalecen los músculos, al aumentar su potencia muscular y mejorar el sprint, con sus cualidades de velocidad y aceleración así como su utilización en la prevención de lesiones musculotendinosas, crea polémica entre entrenadores y estudiosos del tema.

Al respecto encontramos a Eckard, Padua, Hearn, Pexa, \& Frank (2018); Lagos (2019), quienes concuerdan que un aspecto a tener en cuenta está dado principalmente por la dosificación de los ejercicios y los posibles picos de carga que pudieran acarrear una incorrecta planificación de los mismos, lo que pudieran traer consigo lesiones y reducción de los componentes musculoesqueléticos, así como, que los entrenadores y dosificadores deben tener un profundo conocimiento de los factores de rendimiento del fútbol sala, en el cual de manera preponderante abundan las diferentes acciones de intensidad y cambios de ritmo en las velocidades de desplazamiento, constituyendo de hecho uno de los pasos iniciales a tener en cuenta en la programación de los tipos de ejercicios que contengan los programas de entrenamiento, con vistas a alcanzar los valores óptimos y conjuntamente elaborar las estrategias preventivas para evitar las lesiones.

Por otra parte, se cuentan con varios autores y entrenadores que han elaborado programas de entrenamientos en los que preponderantemente se dan experiencia con ejercicios pliométricos, entre los que se destacan, Sadoghi, von Keudell, \& Vavken (2012); Romero, Cuéllar, González, Bastida, Echarri, Gallardo, Paredes (2017); Saiz (2018); Acosta, Cetina, Ramírez, \& Montealegre-Mesa (2019); Vargas (2019) y Reina (2020), quienes corroboran que el ejercicio pliométrico desarrolla la capacidad del deportista de asimilar los diferentes cambios de posición y ritmo en el movimiento, los que mejoran los niveles de adaptabilidad osteomioarticular disminuyendo las lesiones, debido al incremento de la capacidad de trabajo propioceptivo a nivel de las estructuras anatómicas del tren inferior del cuerpo. De igual manera se refieren a los principales programas preventivos reconocidos por su especificidad y otros desarrollados por la Federación Internacional de Fútbol Asociado FIFA. 
Otro aspecto relacionado con la aplicación del trabajo pliométrico que es objeto de divergencias, es el referido al periodo de duración de los mismos y su efectividad, al respecto Sadoghi, von Keudell, \& Vavken (2012) en su estudio determinaron un 85\% de efectividad en los deportistas hombres y un 52\% en las mujeres. También Teixeira et al. (2017) obtuvieron rendimientos significativos en la capacidad de sprint repetidos, así como la utilización de intervalos de alta intensidad, el cual se obtuvo tras cinco semanas de entrenamiento específico con cambios de dirección en los movimientos. De igual manera, Ayala et al. (2017), obtuvieron alentadores resultados en un equipo español, siendo muy eficaz el aumento del rango del movimiento (ROM) de cadera, tanto de flexión como extensión,

realizado tras 8 semanas de intervención con 6 series x 30 segundos de estiramiento activo durante tres días a la semana.

Considerando y reflexionado sobre los aportes precedentes resulta básico resumir algunas ideas generales que forman parte concluyente del análisis realizado:

- Se coincide con Acosta, Cetina, Ramírez, \& Montealegre-Mesa (2019), en que la prevención de lesiones en el jugador de fútbol, es una estrategia de abordaje fundamental, dado que está relacionada con las consecuencias negativas que tiene tanto para su rendimiento deportivo como para la salud del jugador durante toda su carrera deportiva y además trae beneficios en que se acelere el proceso de recuperación funcional del jugador para su incorporación al terreno de juego, con mejores condiciones físicas.

- La aplicación de entrenamientos con ejercicios pliométricos deben estar basados en las particularidades específicas del futsal, de manera que con la planificación de los ejercicios respectivos se obtengan los resultados esperados, relacionados con la elevación del rendimiento de los futbolistas ligado a la prevención de las lesiones, siendo vital para ello el concocimiento de la información sobre cargas mecánicomusculares, tales como, la distancia total recorrida (TD), poder metabólico (MP), potencia metabólica de alta intensidad (HDMI) y la carga dinámica del cuerpo (DBL), aspectos que deben tener en cuenta los entrenadores al aplicar el entrenamiento.

- El principal beneficio que ofrece la pliometría es la prevención de lesiones musculotendinosas; dado que al realizar los ejercicios pliométricos, se fortalece de hecho, toda la musculatura del miembro inferior, además aumentan su potencia muscular donde se desarrolla la fuerza, el equilibrio y la coordinación, también se mejora el sprint, que contiene a cualidades fundamentales del futbol tales como: la aceleración y la velocidad. 
- Dada las limitaciones en el contexto ecuatoriano en cuanto a estudios relacionados con este tema se requiere direccionar la apertura de nuevas investigaciones en esta línea.

- Todo lo anterior nos permite asumir la necesidad de introducir cambios en los modelos y metodologías actuales de entrenamiento en el futsal, los cuales deben basarse en las nuevas actualizaciones de los programas de entrenamiento aplicados actualmente con éxito a nivel internacional.

Según nuestro criterio no solo es determinante las divergencias o polémicas que se han creado en relación a las formas y duración de los entrenamientos con los ejercicios pliométricos, sino, lo realmente importante es saber planificar y dosificar las cargas de entrenamiento en correspondencia con las particularidades del futsal, de manera que se obtenga el rendimiento esperado y teniendo en cuenta qué hacer para alcanzar progresivamente los resultados esperados.

Siguiendo estas ideas y en correspondencia con la sistematización de las diferentes fuentes y teorías consultadas, que constituyen antecedentes potenciales de la investigación que se presenta, se considera trascendente valorar tres puntos de gran jerarquía:

$\checkmark$ Se coincide con los autores precedentes en la importancia que tiene el trabajo pliométrico en la prevención de lesiones, con especial interés en el futsal.

$\checkmark$ El segundo está relacionado con las limitaciones existente en el contexto ecuatoriano, en cuanto al avance de investigaciones que promuevan considerar con mayor profundidad el desarrollo e implementación en la práctica de las teorías que reconocen los beneficios del trabajo pliométrico en la prevención de lesiones en los jugadores de futsal.

$\checkmark$ El tercer punto nos hace concordar con los autores precedentes en la necesidad de contar con un cuerpo de entrenadores motivados, capacitados y competentes capaces de introducir novedosas formas y alternativas de entrenamiento para evitar las posibles lesiones que pueden darse como consecuencia de no emplear el trabajo pliométrico en el futsal.

Es importante significar además que, en el caso del futsal, debido a las propias características de este deporte, ha quedado fundamentado la importancia del trabajo pliométrico para la prevención de lesiones. A esto se une la necesidad de la actualización de los entrenadores, del cuerpo técnico y de los propios jugadores como una acción fundamental en los momentos actuales, no solo para la mejora constante del rendimiento y los resultados deportivos, sino también para la prevención de lesiones durante el entrenamiento y como parte de las competencias. En concordancia con ello, coincidimos 
con Eckard, Padua, Hearn, Pexa, \& Frank (2018), al plantear que los dos principales objetivos del entrenamiento con los ejercicios pliométricos son: optimizar el rendimiento y lograr reducir el número y severidad de las lesiones.

En resumen, en el contexto del futsal, como en otros deportes, se trata de un tema altamente atrayente e importante a considerar como parte del entrenamiento deportivo; su pertinencia y actualidad hacen que sea necesario continuar profundizando en el mismo desde una visión integral del problema objeto de estudio.

\section{Conclusiones}

A modo de conclusión se considera que:

- La RSL (Revisión Sistemática de Literatura), permitió sistematizar la importancia y beneficios que tiene la aplicación del trabajo pliométrico en los procesos de entrenamiento deportivo, como una alternativa eficaz para la prevención de lesiones, la mejora del rendimiento y los resultados deportivos de los jugadores de futsal. Resultando fundamental aperturar nuevas investigaciones en esta línea dentro del contexto ecuatoriano.

- Resulta fundamental promover el desarrollo de procesos de capacitación permanente para lograr la actualización constante de los entrenadores, del cuerpo técnico y de los jugadores en los diferentes niveles, de modo tal que se logre la introducción de estas novedosas formas de entrenamiento.

\section{Bibliografía}

Acosta, N., Cetina, M., Ramírez, J., \& Montealegre-Mesa L.M. (2019). Programas preventivos, una estrategia para el jugador de fútbol. Revisión bibliográfica. Revista Actividad Física y Deporte, 6 (1): 109-128.

Adalid, J. (2014). Propuesta de incorporación de tareas preventivas basadas en métodos propioceptivos en fútbol. Universidad de Granada. Retos, 26, 163-167.

Alfaro, J., Salicetti, F., y Jiménez, J. (2018). Efecto del Entrenamiento Pliométrico en la Fuerza Explosiva en Deportes Colectivos: Un Metaanálisis. Pensar En Movimiento: Revista de Ciencias del Ejercicio y la Salud, 16(1), 27-52. https//dx.doi.org/10.15517/pensarmov.v16i1.27752 
Álvarez, J., Murillo, V., Giménez, L., y Manonelles , P. (2016). Modificación Del Volumen-Intensidad Como Medida Preventiva de Lesiones En Fútbol Sala / Modification of Volume-Intensity as Preventive Measure in Futsal Injuries. Revista Internacional de Medicina y Ciencias de la Actividad Física y del Deporte, 61, 8597. https://doi.org/10.15366/rimcafd2016.61.007

Åman, M., Larsén, K., Forssblad, M., Näsmark, A., Waldén, M., \& Hägglund, M. (2018). A Nationwide Follow-up Survey on the Effectiveness of an Implemented Neuromuscular Training Program to Reduce Acute Knee Injuries in Soccer Players. Orthopaedic. Journal of Sports Medicine, 6(12), 1-10. https://doi.org/10.1177/2325967118813841.

Ayala, F., Calderón, A., Delgado, J., Parra, S., Pomares, C., Hernández, S., \& de Ste Croix, M. (2017). Acute effects of three neuromuscular warm-up strategies on several physical performance measures in football players. PLOS ONE, 12(1), 1-17. https://doi.org/10.1371/journal.pone.0169660.

Ayarra, R., Nakamura, F. Y., Iturricastillo, A., Castillo, D., \& Yanci, J. (2018). Differences in Physical Performance According to the Competitive Level in Futsal Players. Journal of Human Kinetics, 64(1), 275-285. https://doi.org/10.1515/hukin2017-0201

Beato, M., Coratella, G., Schena, F., \& Hulton, A. T. (2017). Evaluation of the external $\&$ internal workload in female futsal players. Biology of Sport, 34(3), 227-231. https://doi.org/10.5114/biolsport.2017.65998.

Bizzini, M., Junge, A., \& Dvorak, J. (2013). Implementation of the FIFA 11+ football warm up program: How to approach and convince the Football associations to invest in prevention. British Journal of Sports Medicine, 47(12), 803-806. https://doi.org/10.1136/bjsports-2012- 092124.

Eckard, T., Padua, D., Hearn, D., Pexa, B., \& Frank, B. (2018). The Relationship Between Training Load and Injury in Athletes: A Systematic Review. Sports Medicine (Auckland, N.Z.), 48(8), 1929-1961. https://doi.org/10.1007/s40279-018-0951-z.

Ejnisman, B., Andreoli, C., De Castro, A., Cohen, M., Bizzini, M., Dvorack, J. \& Barbosa, G. (2016). Shoulder injuries in soccer goalkeepers: review and development of a FIFA 11+ shoulder injury prevention program. Open Access Journal of Sports Medicine, 7, 75-80. https://doi.org/10.2147/oajsm.s97917.

García, F., \& Peña López, J. (2016). Efectos de 8 Semanas de Entrenamiento Pliométrico y Entrenamiento Resistido Mediante Trineo en el Rendimiento de Salto Vertical y Esprint en Futbolistas Amateurs. Kronos: Revista Universitaria de la Actividad Física y el Deporte, 15(2), 1-10. Recuperado de: https://n9.cl/tzc9w 
Hamid, M., Jaafar, Z., \& Ali, A. (2014). Incidence and characteristics of injuries during the 2010 FELDA/FAM national futsal league in Malaysia. PLoS ONE, 9(4), 2-7. https://doi.org/10.1371/journal.pone.0095158

Hewett, T., Myer, G., Ford, K., Paterno, M., \& Quatman, C. (2016). Mechanisms, prediction, and prevention of ACL injuries: Cut risk with three sharpened and validated tools. Journal of Orthopaedic Research, 34(11), 1843-1855. https://doi.org/10.1002/jor.23414.

Karavelioglu, M. B., Harmanci, H., Kaya, M., \& Erol, M. (2016). Effects of Plyometric Training on Anaerobic Capacity and Motor Skills in Female Futsal Players. The Anthropologist, 23(30), 355-360. https://doi.org/10.1080/09720073.2014.11891955.

Lagos, C. (2019). Optimización del rendimiento físico-deportivo en futbol sala femenino: Desde la epidemiología lesional a una propuesta de intervención. Tesis Doctoral Universidad de Vigo. España.

López, M., Vivo, I., Herrero, R., \& Pareja, F. (2019). Preseason Injury Characteristics in Spanish Professional Futsal Players. Journal of Strength and Conditioning Research, 20, 6. https://doi.org/10.1519/jsc.0000000000003419.

Markovic, G, \& Mikulic. P. (2010). Adaptaciones neuro-musculoesqueléticas y de rendimiento al entrenamiento pliométrico de las extremidades inferiores. Magazine Sport Medicine 40(10):859-95. https://doi.org/10.2165/11318370-000000000-00000.

Martinez, L., Herrero, H., Lopez, J., Guillen, P., y Fernandez, T. (2017). Epidemiology of Injuries in the Spanish National Futsal Male Team: A Five-Season Retrospective Study. BMJ Open Sport and Exercise Medicine, 2(1), 4-9. https://doi.org/10.1136/bmjsem-2016-000180.

Mesfin, M., Sangeeta, R. \& Molla D. (2015)- Effects of plyometric training on soccer related physical fitness variables of intercollegiate female soccer players. Turk J Kin, 1(1): 20-24. Recuperado de: https://n9.cl/qs5vf

Muñoz E, \& Duro, P., (2012). El entrenamiento pliométrico en el fútbol sala: ganancias de potencia de salto y de potencia de disparo. Lecturas: Educación Física y Deportes, 17 (169), 1-12. Recuperado de: https://n9.cl/5r51

Mueller, H., Haensel, L., Mithoefer, K., Ekstrand, J., English, B., McNally, S., Orchard, J., Van Dijk, N., Kerkhoffs, G., Schamasch, P., Blottner, D., Swaerd, L., Goedhart, E., y Ueblacker, P. (2013). Terminology and classification of muscle injuries in sport: The Munich consensus statement. British Journal of Sports Medicine, 47(6), 342350. https://doi.org/10.1136/bjsports-2012-091448. 
Naser, N., Ali, A., \& Macadam, P. (2017). Physical and physiological demands of futsal. Journal of Exercise Science \& Fitness, 15(2), 76-80. https://doi.org/10.1016/j.jesf.2017.09.001

Neves da Silva, V. F., Aguiar, S. da S., Sousa, C. V., Sotero, R. da C., Filho, J. M. S., Oliveira, I. Sales, M. M. (2017). Effects of short-term plyometric training on physical fitness parameters in female futsal athletes. Journal of Physical Therapy Science, 29(5), 783-788. https://doi.org/10.1589/jpts.29.783.

Noguera, O \& Gamboa, Y. (2018). Influencia de la pliometría en la velocidad de desplazamiento de las jugadoras de fútbol sala femenino de la Corporación Universitaria Autónoma del Cauca- Corporación Universitaria Autónoma del Cauca. Trabajo de grado para optar al título de profesional en Deporte y Actividad Física. Facultad de Educación Programa de Deporte y Actividad Física. Ciencias Aplicadas al Deporte y la Actividad Física. Recuperado de: https://n9.cl/j5co8

Nouni, R.; Carratala, C.; Orozco, D.; López, A., Asensio, M. \& Gil, V. (2018). Clinical benefit of the FIFA 11 programme for the prevention of hamstring and lateral ankle ligament injuries among amateur soccer players. Injury Prevention, 24(2), 149-154. https://doi.org/10.1136/injuryprev-2016-042267.

Pujals, C., Rubio, V., Marquez, M., Sánchez, I., y Ruiz, R. (2016). Comparative Sport Injury Epidemiological Study on a Spanish Sample of 25 Different Sports. Revista de Psicologia Del Deporte, 25(2), 271-79. Recuperado de: http://hdl.handle.net/10486/677646

Reina, L. (2020). Aplicación del Ejercicio Pliométrico como mecanismo para incrementar la Fuerza Explosiva en el tren inferior en futbolistas del Equipo masculino Sub-16 del Club Deportivo "El Nacional". Trabajo de titulación previo a la obtención del Título de Magister en Entrenamiento Deportivo. Maestría en Entrenamiento Deportivo. Vicerrectorado de Investigación, Innovación y Transferencia Tecnológica. Centro de Posgrado.

Rezaimanesh, D., Amiri, P., y Saidian, S. (2011). The Effect of a 4 Week Plyometric Training Period on Lower Body Muscle EMG Changes in Futsal Players. Procedia Social and Behavioral Sciences, 15, 3138-42. https://doi.org/10.1016/j.sbspro.2011.04.260

Rodríguez-Gómez, G. A., Merchan, J. A., \& Forero, S. A. (2014). Comportamiento de la Fuerza Explosiva, la Agilidad y la Velocidad ante un Calentamiento con Sobrecarga en Futbolistas. Revista de Entrenamiento Deportivo, 28(4).

Romero, B., Cuéllar, A., González, J., Bastida, N., Echarri, E., Gallardo, J., y Paredes, V. (2017). Revisión de los factores de riesgo y los programas de prevención de la lesión 
del ligamento cruzado anterior en fútbol femenino: propuesta de prevención. RICYDE. Revista Internacional de Ciencias del Deporte, 13 (48), 117-138. https://doi.org/10.5232/ricyde2017.04803

Roopchand-Martin S, Lue-Chin P. (2010). Plyometric training improves power and agility in Jamaica's National Netball Team. West Indian Med J. 59(2): 182-187. Recuperado de: https://n9.cl/f0k78

Sadoghi, P., von Keudell, A., \& Vavken, P. (2012). Effectiveness of anterior cruciate ligament injury prevention training programs. JBJS, 94(9), 769-776. https://doi.org/10.2106/JBJS.K.00467.

Sekulic, D., Foretic, N., Gilic, B., Esco, M., Hammami, R., Uljevic, O., Versic, M., \& Spasic, M. (2019). Importance of Agility Performance in Professional Futsal Players; Reliability and Applicability of Newly Developed Testing Protocols. International Journal of Environmental Research and Public Health 16(18), 2-12. https://doi.org/10.3390/ijerph16183246.

Simbaña, A. (2018). Los ejercicios pliométricos en la fuerza explosiva del tren Inferior de la selección de futbol femenino de la Liga Deportiva Parroquial Picaihua. Tesis de Grado. Universidad Técnica de Ambato. Ecuador. Recuperado de: https://n9.cl/90p6

Sinovas, M., Rodríguez, M., \& Cerezal, A. (2020). Epidemiology of Injuries in Young Spanish Soccer Players According to the Playing Positions. Retos, 83, 459-64. Recuperado de: https://url2.cl/46Ycw

Tomalá E, (2020). Programa de ejercicios pliométricos para el desarrollo de la fuerza explosiva en futbolistas de la categoría sub 16. Maestría profesional en Entrenamiento Deportivo. Universidad Estatal de Milagro. UNEMI Ecuador.

Tsang, K. K., \& DiPasquale, A. A. (2011). Improving the Q:H strength ratio in women using plyometric exercises. Journal of Strength and Conditioning Research, 25(10), 2740-2745. https://doi.org/10.1519/JSC.0b013e31820d9e95.

Vargas, A (2019). Pliometría en la prevención de lesiones musculotendinosas en futbolistas profesionales. Club Deportivo el Nacional Quito. Proyecto de Investigación previo a la obtención del título de Licenciada en Terapia Física y Deportiva. Universidad Nacional de Chimborazo. Facultad de Ciencias de la Salud. Carrera de Terapia Física y Deportiva. Recuperado de: https://n9.cl/ndj8

Van Beljstrveldt, A., Van de Port, I., Krist, M., Schmikli, S., Stubbe, J., Frederiks, J., \& Backx, F. (2012). Effectiveness of an injury prevention programme for adult male 
amateur soccer players: a cluster-randomised controlled trial. British Journal of Sports Medicine, 46(16), 1114-1118. https://doi.org/10.1136/bjsports-2012-091277.

Willadsen, E., Zahn, A., \& Durall, C. (2019). What Is the Most Effective Training Approach for Preventing Noncontact ACL Injuries in High School-Aged Female Athletes? Journal of Sport Rehabilitation, 28(1), 94-98. https://doi.org/10.1123/jsr.2017-0055. 
PARA CITAR EL ARTÍCULO INDEXADO.

Arias Granizo, W. G. (2021). Importancia del trabajo pliométrico en la prevención de lesiones en jugadores de fútbol sala: Importancia del trabajo pliométrico en la prevención de lesiones en jugadores de fútbol sala. Ciencia Digital, 5(2), 16-45. https://doi.org/10.33262/cienciadigital.v5i2.1531

\section{Liencia}

El artículo que se publica es de exclusiva responsabilidad de los autores y no necesariamente reflejan el pensamiento de la Revista Ciencia Digital.

El artículo queda en propiedad de la revista y, por tanto, su publicación parcial y/o total en otro medio tiene que ser autorizado por el director de la Revista Ciencia Digital.
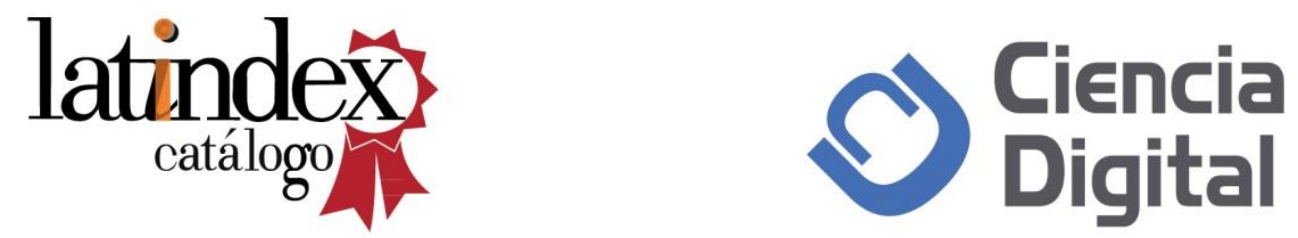